\title{
PRINCIPAL AND ANTIPRINCIPAL SOLUTIONS OF SELFADJOINT DIFFERENTIAL SYSTEMS AND THEIR RECIPROCALS ${ }^{1}$
}

\author{
CALVIN D. AHLBRANDT
}

\begin{abstract}
The concepts of principal and antiprincipal solutions for disconjugate differential systems are applied to the reciprocal and obverse systems. Relationships are obtained between these types of solutions for such systems and their reciprocals.
\end{abstract}

1. Introduction. It has recently been observed [2, Theorem 3.1] that, under certain "variational" assumptions, differential systems of the form

$$
y^{\prime}=G z, \quad z^{\prime}=-F y \quad \text { on }(0, \infty)
$$

are disconjugate in a neighborhood of $\infty$ if and only if the "reciprocal" system

$$
y^{\prime}=F z, \quad z^{\prime}=-G y
$$

is disconjugate in a neighborhood of $\infty$.

From the work of Hartman [5], and Reid [10], it is known that under suitable hypotheses, system (1.1) is disconjugate in a neighborhood of $\infty$ if and only if there exists a principal solution of (1.1) at $\infty$. Consequently, one is led to the study of principal solutions of (1.2) and the relationships between these and related concepts.

Section 2 gives introductory lemmas and definitions of principal, coprincipal, antiprincipal, and anticoprincipal solutions of differential systems. In particular the definition of principal solution differs from that given previously [5], [10], but is shown to be equivalent in certain cases to the definitions of Hartman and Reid. The main results of the paper are given in $\$ 4$. In particular, relationships are obtained between various types of solutions. $\$ 5$ applies these results to scalar second order linear equations.

Received by the editors August 3, 1970.

AMS 1970 subject classifications. Primary 34C10; Secondary 34C10.

Key words and phrases. Selfadjoint differential systems, principal solution, nonprincipal solution, antiprincipal solution, coprincipal solution, anticoprincipal solution, reciprocal system, Reid's obverse system, disconjugate system.

1The research and preparation of this paper was supported in part by the University of Missouri Faculty Improvement Program during the summer of 1970.

Copyright $\odot 1972$ Rocky Mountain Mathematics Consortium 
Matrix notation is used throughout; in particular, matrices of one column are called vectors, all identity matrices are denoted by $E$, and 0 is used indiscriminately for the zero matrix of any dimensions. If $H$ and $K$ are $n \times r$ matrices, or functions, and the symbol $(H ; K)$ is used to denote the $2 n \times r$ partitioned matrix $\left(H^{*} K^{*}\right)^{*}$, where ${ }^{*}$ denotes conjugate transpose. If $H$ and $K$ are $n \times n$ hermitian matrices, we write $H \geqq K[H>K]$ to indicate that $\pi^{*}(H-K) \pi$ is nonnegative [positive] for every unit column vector $\pi$. The symbol $\lambda(H)$ is used to denote the least proper value of an $n \times n$ hermitian matrix, or function, $H$. An $n \times n$ hermitian matrix valued function $H$ on an interval $X$ is said to be nondecreasing, [increasing], if and only if $x_{1}<x_{2}$ implies $H\left(x_{1}\right) \leqq H\left(x_{2}\right) \quad\left[H\left(x_{1}\right)<H\left(x_{2}\right)\right]$; also $H$ is said to be nonnegative on $X$ if $H(x) \geqq 0$ for all $x$ in $X$. Matrix valued functions will be said to have a property of differentiability, continuity, or integrability if and only if each entry of the matrix has that property.

2. Definitions and preliminary material. Suppose that $X$ is a subinterval of the real line and, for each point $x$ in $X$, we have $n \times n$ complex matrices $A(x), B(x)$, and $C(x)$. For future reference, we state the following hypothesis:

(H) The $n \times n$ matrices $B(x)$ and $C(x)$ are hermitian for each point $x$ in $X$, and the functions $A, B$, and $C$ are Lebesgue integrable on arbitrary compact subintervals of $X$.

Consider the differential system

$$
\mathcal{\partial y} y^{\prime}=\mathfrak{2} y,
$$

on $X$, where $\mathcal{J}$ and $\mathfrak{U}$ are $2 n \times 2 n$ complex matrix valued functions defined on $X$ by

$$
\mathcal{J}=\left[\begin{array}{rr}
0 & -E \\
E & 0
\end{array}\right], \text { and } \mathfrak{A}=\left[\begin{array}{rl}
-C & A^{*} \\
A & B
\end{array}\right] .
$$

For $r$ a natural number, a $2 n \times r$ matrix valued function $y$ on $X$ is said to be a solution of (2.1) on $X$ if and only if $y$ is absolutely continuous (a.c.) on arbitrary compact subintervals of $X$ and equation (2.1) is satisfied almost everywhere (a.e.) on $X$. Equivalently, if $y$ is denoted by $(U ; V)$, where $U(x)$ and $V(x)$ are $n \times r$ complex matrices for every $x$ in $X$, then $y$ is a solution of (2.1) on $X$ if and only if $(U ; V)$ is a.c. on arbitrary compact subintervals of $X$ and the equations

$$
U^{\prime}=A U+B V, \quad V^{\prime}=C U-A^{*} V
$$

are satisfied a.e. on $X$. 
Lemma 2.1. Suppose that hypothesis $(\mathbf{H})$ holds and $y_{1}$ and $y_{2}$ are respectively $2 n \times r_{1}$ and $2 n \times r_{2}$ solutions of (2.1) on $X$. Then the $r_{1} \times r_{2}$ complex matrix valued function $y_{1}^{*} \mathcal{2}^{*} y_{2}$ is constant on $X$.

If hypothesis (H) holds, then a $2 n \times n$ solution $y$ of $(2.1)$ is called self-conjoined if and only if the constant function $y^{*} z^{*} y$ is 0 on $X$. If $X_{0}$ is a nondegenerate subinterval of $X$, then system (2.1) is said to be normal on $X_{0}$ if and only if the only $2 n \times 1$ solution of (2.1) of the form $(u ; v)$ with $u$ being the $n \times 1$ zero on $X_{0}$ is the zero solution. System (2.1) is said to be identically normal on $X$ if and only if system (2.1) is normal on every nondegenerate subinterval of $X$.

Lemma 2.2. Suppose that hypothesis $(\mathbf{H})$ holds and $y_{0}$ and $y$ are $2 n \times n$ solutions of (2.1) on $X$ such that $y_{0}{ }^{*} J^{*} y$ is nonsingular. Then, for each point $x$ in $X$, the columns of $y(x)$ are linearly independent. If, in addition, $X_{0}$ is a nondegenerate subinterval of $X$ such that system (2.1) is normal on $X_{0}$, and $y$ is denoted by $(U ; V)$, then the columns of $U$ are linearly independent functions on $X_{0}$.

Lemma 2.3. Suppose that hypothesis $(\mathbf{H})$ holds, and $y_{1}$ and $y_{2}$ are $2 n \times n$ solutions of $(2.1)$ on $X$. Then we have

$$
\mathcal{J}\left(y_{1} y_{2}\right)^{*} \mathcal{J}^{*}\left(y_{1} y_{2}\right)=\left[\begin{array}{ll}
y_{2}^{*} \partial y_{1} & y_{2}^{*} \partial y_{2} \\
y_{1} * \partial^{*} y_{1} & y_{1}^{*} \partial^{*} y_{2}
\end{array}\right]
$$

on $X$. If $y_{2}$ is self-conjoined and the columns of $y_{2}$ are linearly independent on $X$, then there exists a $2 n \times n$ self-conjoined solution $y_{0}$ of (2.1) such that $y_{0}{ }^{*} y^{*}$ is $E$. For any such $y_{0}$, the matrix $\left(y_{0}(x) y_{2}(x)\right)$ is nonsingular at each point $x$ of $X$, and

$$
\left(y_{0} y_{2}\right)^{-1}=\mathcal{J}\left(y_{0} y_{2}\right)^{*} \mathcal{J}^{*}
$$

on $X$.

Lemma 2.4. Suppose that hypothesis $(\mathbf{H})$ holds, and $y_{1}$ and $y_{2}$ are $2 n \times n$ self-conjoined solutions of (2.1) on $X$. Then the following conditions are equivalent.

(i) The constant function $y_{1}{ }^{*} \partial^{*} y_{2}$ is nonsingular.

(ii) If $C_{1}$ and $C_{2}$ are $n \times n$ matrices of complex numbers such that $y_{1} C_{1}+y_{2} C_{2}$ is 0 on $X$, then $C_{1}$ and $C_{2}$ are both zero.

(iii) The $2 n \times 2 n$ solution $\left(y_{1} y_{2}\right)$ of $(2.1)$ on $X$ is fundamental.

Lemma 2.5. Suppose that hypothesis (H) holds, and $y_{0}$ and $y$ are $2 n \times n$ self-conjoined solutions of (2.1) on $X$ such that $y_{0}{ }^{*} \partial^{*} y$ is $E$. Then a $2 n \times n$ function $y_{1}$ is a solution of (2.1) if and only if $y_{1}$ is expressible in the form 


$$
y_{1}=y_{0} C_{0}+y C_{1}
$$

on $X$, where $C_{0}$ and $C_{1}$ are $n \times n$ complex matrices. In particular, if $y_{1}$ is $a 2 n \times n$ solution of (2.1) related to $y_{0}$ and $y$ by (2.4), then $C_{0}$ and $C_{1}$ are uniquely determined as $y^{*} \mathcal{J} y_{1}$ and $y_{0} * \partial^{*} y_{1}$, respectively, and $y_{1}{ }^{*}{ }^{*} y_{1}$ is $C_{0}{ }^{*} C_{1}-C_{1}{ }^{*} C_{0}$.

The results in Lemma 2.3 were known to Radon [9, pp. 292-293]. The solution $y_{0}$ is obtained by letting $y_{1}$ be a $2 n \times n$ solution such that $\left(y_{1} y_{2}\right)$ is fundamental and by determining $C_{1}$ and $C_{2}$ as $n \times n$ constant matrices such that $y_{0}$ defined as $y_{1} C_{1}+y_{2} C_{2}$ has the desired properties. This may be done by observing that the right-hand member of (2.3) is nonsingular, hence $y_{1}^{*} z^{*} y_{2}$ is nonsingular, and one may choose $C_{1}$ as $\left(y_{2}{ }^{*} \partial y_{1}\right)^{-1}$ and $C_{2}$ as $C_{1}{ }^{*} y_{1} * \partial y_{1} C_{1} / 2$.

If $y_{1}$ and $y_{2}$ are $2 n \times n$ solutions of (2.1), the constant $y_{1}^{*} z^{*} y_{2}$ of Lemma 2.1 was referred to by Barrett $[3$, p. 80] as the wronskian in the case of real solutions of differential systems with real coefficients. Lemma 2.4 illustrates that when restricted to self-conjoined solutions, the function $y_{1}^{*} z^{*} y_{2}$ has properties which are analogous to those of the wronskian of solutions of the scalar equation $y^{\prime \prime}+f(t) y=0$. If one considers the set of $2 n \times n$ solutions of (2.1), together with the operations of pointwise addition and right scalar multiplication from the ring $C_{n n}$ of $n \times n$ matrices of complex numbers, then we have the algebraic structure of a right unitary module over $C_{n n}$. See Warner [16, p. 253], for terminology. In this setting, condition (ii) is linear independence of $y_{1}$ and $y_{2}$. For terminology used in condition (iii) of Lemma 2.4, see [6, p. 47]. Moreover, conditions (ii) and (iii) are equivalent without the assumption that $y_{1}$ and $y_{2}$ are self-conjoined, and it can be shown that this module has well-defined dimension of 2.

Suppose that $c$ is either (i) a point of $X$, (ii) the infimum of $X$ (possibly $-\infty$ ) or (iii) the supremum of $X$ (possibly $+\infty$ ). If $c$ is a point of $X$, then by the phrase "neighborhood of $c$ ", we mean a subinterval $X_{0}$ of $X$ such that $c$ is in $X_{0}$, and $X_{0}$ is open in the relative topology of $X$. If $c$ is the infimum [supremum] of $X$ and $c$ is not in $X$, then a "neighborhood of $c$ " is understood to be an interval of the form $(c, b)[(b, c)]$ for some $b$ in $X$. For the purposes of the following definitions, suppose that hypothesis (H) holds. A $2 n \times n$ solution $(U ; V)$ of $(2.1)$ is said to be principal at $c$ if and only if $(U ; V)$ is selfconjoined and there exists a $2 n \times n$ self-conjoined solution $\left(U_{0} ; V_{0}\right)$ of (2.1) such that $U_{0}{ }^{*} V-V_{0}{ }^{*} U$ is nonsingular, $U_{0}$ is nonsingular in a neighborhood of $c$, and we have 


$$
\lim _{x \rightarrow c} U_{0}^{-1}(x) U(x)=0 .
$$

A $2 n \times n$ solution $(U ; V)$ of $(2.1)$ is said to be antiprincipal at $c$ if and only if $(U ; V)$ is self-conjoined and there exists a $2 n \times n$ self-conjoined solution $\left(U_{0} ; V_{0}\right)$ of $(2.1)$ such that $U_{0}{ }^{*} V-V_{0}{ }^{*} U$ is nonsingular, $U_{0}$ is nonsingular in a neighborhood of $c$, and

$$
\lim _{x \rightarrow c} U_{0}^{-1}(x) U(x)=E .
$$

Consider the system “obverse" to (2.2), (see Reid $[13, \S 2]$ ),

$$
U^{\prime}=-A^{*} U+C V, \quad V^{\prime}=B U+A V,
$$

on $X$. Under hypothesis $(\mathbf{H})$, we refer to a $2 n \times n$ solution $(U ; V)$ of (2.1) as being a coprincipal solution of (2.1) at $c$ if and only if the solution $\left(U_{1} ; V_{1}\right)$ of $(2.7)$ defined by $\left(U_{1} ; V_{1}\right)=(V ; U)$ is a principal solution of $(2.7)$ at $c$. Similarly, a $2 n \times n$ solution $(U ; V)$ of $(2.1)$ will be said to be an anticoprincipal solution of (2.1) at $c$ if and only if $\left(U_{1} ; V_{1}\right)$ as defined above is an antiprincipal solution of $(2.7)$ at $c$. Equivalently, a $2 n \times n$ solution $(U ; V)$ of $(2.1)$ may be defined as coprincipal [anticoprincipal] at $c$ if and only if the function $\left(U_{2} ; V_{2}\right)$ defined by $\left(U_{2} ; V_{2}\right)=(V ;-U)$ is principal [antiprincipal] at $c$ for the system "reciprocal" $[2, \$ 2]$ to $(2.1)$ defined by

$$
U^{\prime}=-A^{*} U-C V, \quad V^{\prime}=-B U+A V,
$$

on $X$.

Notice that if $(U ; V)$ is a $2 n \times n$ solution of $(2.1)$ which is principal at $c$, then there exists a $2 n \times n$ self-conjoined solution $\left(U_{0} ; V_{0}\right)$ of (2.1) such that $U_{0}{ }^{*} V-V_{0}{ }^{*} U$ is $E$, while $U_{0}$ is nonsingular in a neighborhood of $c$, and relation (2.5) holds. Also, in the definition of an antiprincipal solution, the solution $\left(U_{0} ; V_{0}\right)$ can be chosen so that $U_{0}{ }^{*} V-V_{0}{ }^{*} U$ is $E$, if, instead of assuming that relation (2.6) holds, we have the existence of a nonsingular $n \times n$ matrix $M$ such that $\lim _{c} U_{0}^{-1} U$ is $M$. Observe that if $c$ is a point of $X$, then the $2 n \times n$ solution $(U(, c) ; \quad V(, c)) \quad\left[\left(U_{0}(, c) ; \quad V_{0}(, c)\right)\right]$ which satisfies $(U(c, c) ; V(c, c))=(0 ; E)\left[\left(U_{0}(c, c) ; V_{0}(c, c)\right)=(E ; 0)\right]$ is principal and anticoprincipal [antiprincipal and coprincipal] at $c$.

TheOREM 2.1. Suppose that hypothesis $(\mathbf{H})$ holds and $y$, denoted by $(U ; V)$, is a $2 n \times n$ solution of $(2.1)$ which is principal at $c$. If $y_{0}$, denoted by $\left(U_{0} ; V_{0}\right)$, is a $2 n \times n$ self-conjoined solution of $(2.1)$ such that $y_{0}{ }^{*} \mathcal{J}^{*} y$ is nonsingular, $U_{0}$ is nonsingular in a neighborhood of c, and relation (2.5) holds, then $y_{0}$ is antiprincipal at $c$. 
Suppose that $C_{0}$ and $C_{1}$ are defined as $E$ and $y^{*} \partial_{y_{0}}$, respectively. Let $y_{1}$, denoted by $\left(U_{1} ; V_{1}\right)$, be defined by equation (2.4) on $X$. Then $y_{1}$ is self-conjoined, $y_{1}{ }^{*} z^{*} y_{0}$ is nonsingular, and in a neighborhood of $c$, we have

$$
U_{0}^{-1} U_{1}=E+U_{0}^{-1} U C_{1}
$$

and hence, $\lim _{c} U_{0}{ }^{-1} U_{1}$ is $E$. Thus $U_{1}$ is nonsingular in a neighborhood of $c$ and $\lim _{c} U_{1}^{-1} U_{0}$ is $E$.

Corollary. Suppose that hypothesis $(\mathbf{H})$ holds and $y$, denoted by $(U ; V)$, is a $2 n \times n$ solution of $(2.1)$ which is coprincipal at $c$. If $y_{0}$, denoted by $\left(U_{0} ; V_{0}\right)$ is a $2 n \times n$ self-conjoined solution of $(2.1)$ such that $y_{0}{ }^{*} z^{*} y$ is nonsingular, $V_{0}$ is nonsingular in a neighborhood of c, and $\lim _{c} V_{0}{ }^{-1} V$ is 0 , then $y_{0}$ is anticoprincipal at $c$.

Theorem 2.2. Suppose that hypothesis (H) holds, system (2.1) is identically normal on $X$, and $B$ is nonnegative on $X$. If $y$, denoted by $(U ; V)$, is a $2 n \times n$ solution of $(2.1)$ on $X$ which is principal at $c$, and $y_{1}$, denoted by $\left(U_{1} ; V_{1}\right)$, is a $2 n \times n$ solution of $(2.1)$ such that $y_{1}{ }^{*} \partial^{*} y$ is nonsingular, then $U_{1}$ and $U$ are nonsingular in a deleted neighborhood of $c$, and $\lim _{c} U_{1}^{-1} U$ is 0 .

The word deleted is redundant unless $c$ is in $X$. Suppose that $y_{0}$, denoted by $\left(U_{0} ; V_{0}\right)$, is a $2 n \times n$ self-conjoined solution such that $U_{0}$ is nonsingular in a neighborhood of $c, y_{0}{ }^{*} \partial^{*} y$ is $E$, and relation (2.5) holds. From Lemma 2.5, there exist $n \times n$ matrices $C_{0}$ and $C_{1}$ such that equation (2.4) holds on $X$. Also, $C_{0}$ is nonsingular. The nonsingularity of $U_{1}$ in a neighborhood of $c$ follows from the result that $U_{0}^{-1}(x) U_{1}(x) \rightarrow C_{0}$, as $x \rightarrow c$. From Lemma 2.2, we are assured that the columns of $U$ are linearly independent functions on $X$. The sturmian separation theorem for systems assures that $U$ is nonsingular in a deleted neighborhood $X_{1}$ of $c$. For the case in which $B$ is positive definite on $X$, the sturmian separation theorem may be found in $[8$, p. 64 , Corollary 1$]$, and in the generality needed here, in [14, Lemma VII 7.1]. For each constant $n$-vector $\pi$, we have

$$
U^{-1}(x) U_{1}(x) \pi=U^{-1}(x) U_{0}(x) C_{0} \pi+C_{1} \pi
$$

for each $x$ in $X_{1}$. Thus, for each $x$ in $X_{1}$, we have

$$
\min _{\pi^{*} \pi=1}\left|U^{-1}(x) U_{1}(x) \pi\right| \geqq \min _{\pi^{*} \pi=1}\left|U^{-1}(x) U_{0}(x) C_{0} \pi\right|-\max _{\pi^{*} \pi=1}\left|C_{1} \pi\right| .
$$

Suppose that for each $x$ in $X_{1}$, the unit vector $\pi(x)$ is such that $\min \left|U^{-1}(x) U_{0}(x) C_{0} \pi\right|=\left|U^{-1}(x) U_{0}(x) C_{0} \pi(x)\right|$. Let $\eta(x)$ be $C_{0} \pi(x)$, and $\zeta(x)=\eta(x) / \eta(x) \mid$, for $x$ in $X_{1}$. Then 


$$
\left|U^{-1}(x) U_{0}(x) \eta(x)\right|=|\eta(x)|\left|U^{-1}(x) U_{0}(x) \zeta(x)\right|,
$$

and we have $1=|\pi(x)| \leqq\left|C_{0}^{-1}\right| \eta(x) \mid$, and $|\eta(x)| \geqq\left|C_{0}^{-1}\right|^{-1}$, where $|C|=\left(\sum_{i=1}^{n}\left|C_{i j}\right|^{2}\right)^{1 / 2}$. Thus

$\min _{\pi^{*} \pi=1}\left|U^{-1}(x) U_{1}(x) \pi\right| \geqq\left(\min _{\pi^{*} \pi=1}\left|C_{0}^{-1}\right|^{-1} \cdot\left|U^{-1}(x) U_{0}(x) \pi\right|\right)-\max _{\pi^{*} \pi=1}\left|C_{1} \pi\right|$,

where $\min _{\pi^{*} \pi=1}\left|U^{-1}(x) U_{0}(x) \pi\right| \rightarrow \infty$, as $x \rightarrow c$. Thus

$$
\max _{\pi^{*} \pi=1}\left|U_{1}^{-1}(x) U(x) \pi\right| \rightarrow 0
$$

as $x \rightarrow c$, (see [10, p. 153], and $U_{1}^{-1}(x) U(x) \rightarrow 0$, as $x \rightarrow c$.

Corollary. Suppose that hypothesis (H) holds, system (2.7) is identically normal on $X$, either $C(x)$ is nonnegative for each $x$ in $X$, or nonpositive for each $x$ in $X$. If $(U ; V)$ is $a 2 n \times n$ solution of $(2.1)$ which is coprincipal at $c$ and $\left(U_{1} ; V_{1}\right)$ is a $2 n \times n$ solution of $(2.1)$ such that $U_{1}^{*} V-V_{1}{ }^{*} U$ is nonsingular, then $V_{1}$ and $V$ are nonsingular in a deleted neighborhood of $c$ and we have $\lim _{x \rightarrow c} V_{1}^{-1}(x) V(x)=0$.

3. Equivalent formulations for the identically normal case. Distinct points, $a$ and $b$, of $X$ are said to be conjugate relative to (2.1) if there exists a $2 n \times 1$ solution $(u ; v)$ of $(2.1)$ such that $u(a)=0=u(b)$ holds, and $u$ is not the zero function on $(a, b)$. For $X_{0}$ a nondegenerate subinterval of $X$, we say that $(2.1)$ is disconjugate on $X_{0}$ if and only if $X_{0}$ contains no pairs of conjugate points.

LEMмa 3.1. Suppose that hypothesis $(\mathbf{H})$ holds, $X_{0}$ is a subinterval of $X$ and $(U ; V)$ is a $2 n \times n$ self-conjoined solution of (2.1) for which $U$ is nonsingular on $X_{0}$. If $a$ is in $X_{0}$, and $\left(U_{0}(x) ; V_{0}(x)\right)$ is defined at each point $x$ of $X_{0}$ by the relations,

$$
\begin{aligned}
& U_{0}(x)=-U(x) \int_{a}^{x} U^{-1}(t) B(t) U^{*-1}(t) d t, \\
& V_{0}(x)=-U^{*-1}(x)-V(x) \int_{a}^{x} U^{-1}(t) B(t) U^{*-1}(t) d t,
\end{aligned}
$$

then $\left(U_{0} ; V_{0}\right)$ is a $2 n \times n$ self-conjoined solution of (2.1) such that $U_{0} * V-V_{0}^{*} U$ is $E$.

TheOREM 3.1. Suppose that hypothesis $(\mathbf{H})$ holds, B is nonnegative on $X$, and system (2.1) is identically normal on X. Suppose also that $c$ is $\sup X$, and $c$ is not in $X$. Let $(U ; V)$ be a $2 n \times n$ self-conjoined solution of (2.1) for which $U$ is nonsingular on $[a, c)$. Then the function $S(, a ; U)$, whose value at each point $x$ of $[a, c)$ is $\int_{a}^{x} U^{-1}(t) B(t) U^{*-1}(t) d t$, is nonsingular on $(a, c)$ and 
(i) $(U ; V)$ is principal at $c$ if and only if $\lim _{c} S^{-1}(, a$; U) is 0 ,

(ii) $(U ; V)$ is antiprincipal at $c$ if and only if there exists a nonsingular matrix $M$ of complex numbers such that $\lim _{c} S(, a ; U)$ is $M$.

Suppose that $b$ is a point of $(a, c)$, and $\pi$ is an $n$-vector such that $S(b, a ; U) \pi$ is 0 . Let $(u ; v)$ be defined as $\left(U_{0} \pi ; V_{0} \pi\right)$ on $[a, b]$, where $\left(U_{0} ; V_{0}\right)$ is defined as in Lemma 3.1. Then $u(a)=0=u(b)$ holds, and $u$ is 0 on $[a, b]$, since $a$ and $b$ cannot be conjugate [11, Theorem 5.2 , p. 678]. From normality, $v$ must be 0 on $[a, b]$, and we have $0=V_{0}(b) \pi=-U^{*-1}(b) \pi$, and $\pi$ is 0 . Conclusion (i) follows from Lemma 3.1 and Theorem 2.2 upon noting that $S^{-1}(, a ; U)$ is $-U_{0}^{-1} U$ on $(a, c)$. If $\lim _{c} S(, a ; U)$ is a nonsingular matrix $M$, then $\left(U_{0} ; V_{0}\right)$, defined as above, is such that $\lim _{c} U_{0}^{-1} U$ is $-M^{-1}$ and $(U ; V)$ is antiprincipal at $c$. Conversely, suppose that $(U ; V)$ is antiprincipal at $c$, and $\left(U_{1} ; V_{1}\right)$ is a $2 n \times n$ self-conjoined solution of $(2.1)$ such that $U_{1}{ }^{*} V-V_{1}^{*} U$ is nonsingular, $U_{1}$ is nonsingular in a neighborhood of $c$ and $\lim _{c} U_{1}{ }^{-1} U$ is $E$. Suppose that $C_{0}$ and $C_{1}$ are $n \times n$ constant matrices such that $U_{1}$ is $U_{0} C_{0}+U C_{1}$ on $[a, c)$. Such constants exist as a consequence of Lemma 2.5 , and furthermore, $C_{0}$ is nonsingular. Thus $U^{-1} U_{1}$ is $-\mathrm{S}(, a ; U) C_{0}+C_{1}$ and $\lim _{c} \mathrm{~S}(, a ; U)$ exists. However, $S(, a ; U)$ is hermitian positive definite, and increasing on $(a, c)$; hence $\lim _{c} S(, a ; U)$ is positive definite.

Conollary. Suppose that hypothesis $(\mathbf{H})$ holds, $C$ is nonnegative on $X$, or $C$ is nonpositive on $X$, system (2.7) is identically normal, $c$ is $\sup X$, and $c$ is not in $X$. Let $(U ; V)$ be $a 2 n \times n$ self-conjoined solution of (2.1) for which $V$ is nonsingular on $[a, c)$. Then the function $\mathrm{S}_{0}(, a ; V)$, whose value at each point $x$ of $[a, c)$ is $\int_{a}^{x} V^{-1}(t) C(t) V^{*-1}(t) d t$, is nonsingular on $(a, c)$, and

(i) $(U ; V)$ is coprincipal at $c$ if and only if $\lim _{c} \mathrm{~S}_{0}{ }^{-1}(, a ; V)$ is 0 ,

(ii) $(U ; V)$ is anticoprincipal at $c$ if and only if there exists a nonsingular matrix $M$ of complex numbers such that $\lim _{c} \mathrm{~S}_{0}(, a ; V)$ is $M$.

The condition $\lim _{c} S^{-1}(, a ; U)=0$ of (i) of Theorem 3.1 is equivalent under those hypotheses to saying that all proper values of $S(, a ; U)$ are unbounded on $(a, c)$. The latter condition was used as the definition of a principal solution by Hartman [5, p. 28]. The existence of $\lim _{c} S(, a ; U)$ is equivalent to saying that all proper values of $S(, a ; U)$ are bounded. Hartman called such a solution nonprincipal, and subsequently Tomastik [15, p. 62], changed that terminology to antiprincipal. In Reid's definition of principal solutions for more general settings [12, p. 408], an assumption is always 
made that $(U ; V)$ has $U$ nonsingular in a neighborhood of $c$. Observe that if $A, B$, and $C$ are all 0 on $X$, then $(0 ; E)$ is a $2 n \times n$ solution of (2.1) which is principal at every point of $X$, and at inf $X$, and $\sup X$, according to the definition used here. Thus the definitions differ in the abnormal cases. Reid did not make the assumption that a principal solution was self-conjoined, but under the hypotheses of the following theorem, the definitions agree [10, p. 159].

Theorem 3.2 [11, p. 677]. Suppose that hypothesis $(\mathbf{H})$ holds, B is nonnegative on $X$, and system (2.1) is identically normal on $X$. Suppose also that $c$ is sup $X$, and $c$ is not in X. Then, system (2.1) is disconjugate in a neighborhood of $c$ if and only if there exists a $2 n \times n$ solution of $(2.1)$ which is principal at $c$.

Theonem 3.3. Under the hypotheses of Theorem 3.2, system (2.1) is disconjugate in a neighborhood of $c$ if and only if there exists a $2 n \times n$ solution of $(2.1)$ which is antiprincipal at $c$.

If system (2.1) is disconjugate in a neighborhood of $c$, then there exists a principal solution and due to the definition and Theorem 2.1 , there exists an antiprincipal solution. Conversely, if $(U ; V)$ is antiprincipal at $c$, then $(U ; V)$ is self-conjoined with $U$ nonsingular in a neighborhood of $c$, and system (2.1) is disconjugate in a neighborhood of $c$ [11, Theorem 5.2, p. 678].

\section{Relationships between principal and coprincipal solutions.}

LEMMA 4.1. If hypothesis (H) holds, $c$ is $\sup X$, and $(U ; V)$ is a $2 n \times n$ self-conjoined solution of (2.1) such that $U$ and $V$ are nonsingular on $[a, c)$, then we have the identity

$$
\begin{aligned}
\int_{a}^{x} U^{-1} B U^{*-1} d t+U^{-1}(x) V^{*-1}(x) \\
\quad=\int_{a}^{x} V^{-1}(-C) V^{*-1} d t+U^{-1}(a) V^{*-1}(a),
\end{aligned}
$$

for $a \leqq x<c$.

Indeed, the lemma follows upon differentiation of both members of (4.1).

THeOREM 4.1. Suppose that hypothesis $(\mathbf{H})$ holds, $B$ is nonnegative on $X, C$ is nonpositive on $X$, and $c$ is $\sup X$, where $\sup X$ is not in $X$. Suppose also that systems (2.1) and (2.8) are identically normal on $X$, and for $D$ a fundamental solution of $D^{\prime}=A D$, we have the condition 


$$
\pi^{*}\left(\int_{a}^{x} D^{-1} B D^{*-1} d t\right) \pi \rightarrow \infty, \quad \text { as } x \rightarrow \infty
$$

for every constant unit vector $\pi$. If $(U ; V)$ is a $2 n \times n$ solution of (2.1) which is principal at $c$, then $(U ; V)$ is coprincipal at $c$.

For $D$ as in the hypotheses of Theorem 4.1, define $Y$ and $Z$ by $Y=D^{-1} U$ and $Z=D^{*} V$ on $X$. Let $G$ and $F$ be defined by $G=D^{-1} B D^{*-1}$ and $F=-D^{*} C D$, respectively. Then $(Y ; Z)$ is a $2 n \times n$ self-conjoined solution of the system

$$
Y^{\prime}=G Z, \quad Z^{\prime}=-F Y, \quad \text { on } X .
$$

Suppose that $U$ is nonsingular on $\left[a_{1}, c\right)$ and $W$ is defined as $Z Y^{-1}$ on $\left[a_{1}, c\right)$. Then $W$ is an $n \times n$ hermitian solution of $W^{\prime}=$ $-F-W G W$, on $\left[a_{1}, c\right)$, and from the normality assumption on system (2.8) together with the nonnegativeness of $F$, we conclude that $a_{1} \leqq x_{1}<x_{2}<c$ implies that $\int_{x_{1}}^{x_{2}} F(t) d t$ is positive definite, (see, for example [1, Lemma 2.1, p. 274]). Thus, $W$ is decreasing on $\left[a_{1}, c\right)$, and each proper value of $W$ is decreasing on $\left[a_{1}, c\right)$. Therefore, each proper value of $W$ can have at most one zero on $\left[a_{1}, c\right)$, and due to finite dimensionality, $W$ is nonsingular in a neighborhood of $c$. Thus $Z$, and consequently $V$, is nonsingular in a neighborhood of $c$.

Now suppose that $U$ and $Y$ are nonsingular on $[a, c)$. Then we have

$$
U^{-1} V^{*-1}=Y^{-1} Z^{*-1}=Z^{-1} W Z^{*-1}, \text { on }[a, c) \text {. }
$$

The assumption that $(U ; V)$ is principal at $c$ implies that for each constant unit vector $\pi$, we have $\pi^{*}\left(\int_{a}^{x} U^{-1} B U^{*-1} d t\right) \pi \rightarrow \infty$, as $x \rightarrow \infty$. The theorem will follow from the lemma once it is shown that $W$ is positive definite on $[a, c)$. For $W_{1}$ defined as $W^{-1}$ on $[a, c)$, it follows that $W_{1}$ is an $n \times n$ hermitian solution of $W_{1}^{\prime}=G+W_{1} F W_{1}$ on $[a, c)$. Hence, $W_{1}$ is increasing and $\pi^{*} W_{1}(x) \pi \rightarrow \infty$, as $x \rightarrow c$, for each constant unit vector $\pi$. Therefore, $\lambda\left(W_{1}(x)\right) \rightarrow \infty$, as $x \rightarrow c$, and $W_{1}$ is positive definite in a neighborhood of $c$. Since the proper values of $W_{1}$ are continuous and never zero on $[a, c)$, we conclude that $W_{1}$, and hence $W$, is positive definite on $[a, c)$.

Reversing the roles of systems (2.1) and (2.8) produces the following result.

Corollary. Suppose that hypothesis $(\mathbf{H})$ holds, B is nonnegative and $C$ is nonpositive on $X$, and $c$ is $\sup X$, where $\sup X$ is not in $X$. Suppose also that systems (2.1) and (2.8) are identically normal on $X$, and for $D$ a fundamental solution of $D^{\prime}=A D$, we have the condition $\pi^{*}\left(\int_{a}^{x} D^{*} C D d t\right) \pi \rightarrow-\infty$, as $x \rightarrow c$, for every constant unit 
vector $\pi$. If $(U ; V)$ is a $2 n \times n$ solution of $(2.1)$ which is coprincipal at $c$, then $(U ; V)$ is principal at $c$.

Lemмa 4.2. Suppose that the hypotheses of Theorem 4.1 hold, and $(U ; V)$ is $a 2 n \times n$ solution of $(2.1)$ which is antiprincipal at $c$. Then $U$ and $V$ are nonsingular in a neighborhood of $c$ and $\lim _{c} U^{-1} V^{*-1}$ is 0 .

Let $\left(U_{2} ; V_{2}\right)$ be defined by the equation

$$
\begin{aligned}
& U_{2}(x)=U(x) \int_{x}^{c} U^{-1} B U^{*-1} d t, \\
& V_{2}(x)=-U^{*-1}(x)+V(x) \int_{x}^{c} U^{-1} B U^{*-1} d t,
\end{aligned}
$$

for $x$ in a neighborhood of $c$. Then $\left(U_{2} ; V_{2}\right)$ is a self-conjoined solution of (2.1) with $\lim _{c} U^{-1} U_{2}=0$, and $\lim _{c}\left(V^{-1} V_{2}+V^{-1} U^{*-1}\right)=0$. Since $U_{2}{ }^{*} V-V_{2}{ }^{*} U$ is nonsingular, it follows that $\left(U_{2} ; V_{2}\right)$ is principal at $c$, and, hence coprincipal at $c$ from Theorem 4.1. Therefore, $\lim _{c} V^{-1} V_{2}$ is 0 and the conclusion of the lemma follows.

Theorem 4.2. Suppose that the hypotheses of Theorem 4.1 hold. Then a $2 n \times n$ solution of (2.1) is antiprincipal at $c$ if and only if it is anticoprincipal at $c$.

Suppose that $(U ; V)$ is a $2 n \times n$ solution of $(2.1)$ which is anticoprincipal at $c$. Application of part (ii) of the corollary to Theorem 3.1 assures that the right-hand member of equation (4.1) has a limit. Since the term $U^{-1}(x) V^{*-1}$ is hermitian and positive definite, the hermitian term $\int_{a}^{x} U^{-1} B U^{*-1} d t$ is bounded above, and consequently has a limit, since it is nondecreasing. Application of part (ii) of Theorem 3.1 assures that $(U ; V)$ is antiprincipal at $c$. The converse follows in a similar manner upon applying Lemmas 4.1 and 4.2.

Reversing the roles of systems (2.1) and (2.8) produces the following corollary.

Corollary 1. Suppose that the hypotheses of the corollary to Theorem 4.1 hold. Then the conclusion of Theorem 4.2 holds.

Corollary 2. Suppose that the hypotheses of Theorem 4.1, or of the corollary to Theorem 4.1, hold and $(U ; V)$ is a $2 n \times n$ solution of (2.1) which is coprincipal [principal] at c. Then there exists a point $a$ in $X$, together with a constant unit vector $\pi$, such that $U$ and $V$ are nonsingular on $[a, c)$, and

$$
\pi^{*}\left(\int_{a}^{x} U^{-1} B U^{*-1} d t\right) \pi \rightarrow \infty, \quad \text { as } x \rightarrow c,
$$




$$
\left[\pi^{*}\left(\int_{a}^{x} V^{-1} C V^{*-1} d t\right) \pi \rightarrow-\infty \text {, as } x \rightarrow c\right]
$$

Theorem 4.3. Suppose that the hypotheses of Theorem 4.1, or the hypotheses of the corollary to Theorem 4.1 hold, and system (2.1) is disconjugate in a neighborhood of $c$. Then there exist $2 n \times n$ selfconjoined solutions $y$ and $y_{0}$ of (2.1) for which $y$ is principal and coprincipal at $c$ and $y_{0}$ is antiprincipal and anticoprincipal at $c$ such that every $2 n \times r$ solution $y_{1}$ of (2.1) is uniquely expressible in the form (2.4), where $C_{0}$ and $C_{1}$ are $n \times$ r complex matrices.

Indeed, under the hypotheses of Theorem 4.1, there exists a $2 n \times n$ solution $y$ which is principal at $c$ due to Theorem 3.1. Application of Theorem 4.1 implies that $y$ is also coprincipal at $c$. It follows from the definition of $y$ being principal at $c$ that there exists a $2 n \times n$ solution $y_{0}$ of (2.1) which satisfies the conditions imposed in Theorem 2.1. Consequently $y_{0}$ is antiprincipal at $c$, and it follows from Theorem 4.2 that $y_{0}$ is anticoprincipal at $c$. Application of Lemma 2.4 completes the proof in this case. The case in which the hypotheses of the corollary to Theorem 4.1 hold follows in a similar manner. Observe that in both cases $y_{0}$ may be chosen so that $y_{0}{ }^{*} y$ is $E$.

5. An application to second order selfadjoint scalar equations. Let $c$ be an extended real number such that $0<c \leqq \infty$ holds, and suppose that $r$ and $f$ are continuous on $(0, c)$ with $r$ positive and $f$ of fixed sign on $(0, c)$. One may consider the differential equation

$$
\left(y^{\prime} / r\right)^{\prime}+f y=0,
$$

on $(0, c)$, and the "reciprocal" equation $[4$, p. 558]

$$
\left(y^{\prime} / f\right)^{\prime}+r y=0,
$$

on $(0, c)$. A differentiable function $y$ on $(0, c)$ is a solution of $(5.1)$ on $(0, c)$ if and only if $y^{\prime} / r$ is a solution of (5.2). A pair of solutions $y_{1}$ and $y_{2}$ of $(5.1)$ on $(0, c)$ is linearly independent on $(0, c)$ if and only if $y_{1}{ }^{\prime}$ and $y_{2}{ }^{\prime}$ are linearly independent on $(0, c)$. A solution $y$ of (5.1) is principal [coprincipal] at $c$ if and only if $y$ is real valued on $(0, c)$, and there exists a real solution $y_{0}$ such that $y$ and $y_{0}$ are linearly independent, $y_{0}$ is nonzero in a neighborhood of $c$, and $\lim _{c} y / y_{0}\left[\lim _{c} y^{\prime} / y_{0}^{\prime}\right]$ exists and is 0 .

Theorem 5.1. Suppose that $r$ and $f$ are continuous positive real valued functions on $(0, c)$ and $\int_{a}^{x} r(t) d t \rightarrow \infty$, or $\int_{a}^{x} f(t) d t \rightarrow \infty$, as $x \rightarrow c$. Then $\phi$ is a solution of $(5.1)$ on $(0, c)$ which is principal at $c$ if and only if $\phi$ is a solution of $(5.1)$ on $(0, c)$ which is coprincipal at $c$. 
Notice that if $\int_{a}^{c} r(t) d t$ and $\int_{a}^{c} f(t) d t$ both exist, the conclusion of Theorem 5.1 does not follow. Indeed, let $c$ be $\pi$ and consider the solution sin of $y^{\prime \prime}+y=0$ on $(0, \pi)$ which is principal at $\pi$ and anticoprincipal at $\pi$. Examples may also be provided on an infinite interval by transforming this one.

Theorem 5.1 follows from Corollary 2 to Theorem 4.2 upon noting that the possibilities in conditions (i) and (ii) of Theorem 3.1 (or its corollary) exhaust the cases when $n$ is 1 . The question as to whether the converse of Theorem 4.1 is true in general remains open.

Corollary. Suppose that $r$ and $f$ are as in Theorem 5.1. Consider two real linearly independent solutions $y_{1}$ and $y_{2}$ of $(5.1)$ on $(0, c)$ which are nonzero in a neighborhood of $c$. Then $\lim _{c} y_{1} / y_{2}$ exists and is 0 if and only if $\lim _{c} y_{1}{ }^{\prime} / y_{2}{ }^{\prime}$ exists and is 0 . Also, $\lim _{c} y_{1} / y_{2}$ exists and is nonzero if and only if $\lim _{c} y_{1}{ }^{\prime} / y_{2}{ }^{\prime}$ exists and is nonzero.

The word coprincipal has been used in this section for what Leighton [7, p. 267] called a focal point solution. The above corollary extends Lemmas 2.5 and 2.6 of Leighton [7, pp. 261-262]. The behavior illustrated in Lemma 4.1 of Leighton [7, pp. 267-268] is expressing a basic property of a principal solution of (5.2), i.e., a coprincipal solution of (5.1).

\section{REFERENCES}

1. Calvin D. Ahlbrandt, Disconjugacy criteria for self-adjoint differential systems, J. Differential Equations 6 (1969), 271-295. MR 39 \#5855.

2. _ Equivalent boundary value problems for self-adjoint differential systems, J. Differential Equations 9 (1971), 420-435.

3. John H. Barrett, Matrix systems of second order differential equations, Portugal. Math. 14 (1956), 79-89. MR 18, 211.

4. — Disconjugacy of second-order linear differential equations with nonnegative coefficients, Proc. Amer. Math. Soc. 10 (1959), 552-561. MR $21 \# 7329$.

5. Philip Hartman, Self-adjoint, non-oscillatory systems of ordinary, second order, linear differential equations, Duke Math. J. 24 (1957), 25-35. MR 18, 576.

6. —_ Ordinary differential equations, Wiley, New York, 1964. MR 30 $\# 1270$.

7. Walter Leighton, Principal quadratic functionals, Trans. Amer. Math. Soc. 67 (1949), 253-274. MR 11, 603.

8. Marston Morse, A generalization of the Sturm separation and comparison theorems in $n$-space, Math. Ann. 103 (1930), 52-69.

9. Johann Radon, Zum Problem von Lagrange, Abh. Math. Sem. Univ. Hamburg 6 (1928), 273-299.

10. William T. Reid, Principal solutions of non-oscillatory self-adjoint linear differential systems, Pacific J. Math. 8 (1958), 147-169. MR 20 \#4682. 
11. - Riccati matrix differential equations and non-oscillation criteria for associated linear differential systems, Pacific J. Math. 13 (1963), 665-685. MR 27 \#4991.

12. — Principal solutions of nonoscillatory linear differential systems, J. Math. Anal. Appl. 9 (1964), 397-423. MR 29 \#6110.

13. - Monotoneity properties of solutions of Hermitian Riccati matrix differential equations, SIAM J. Math. Anal. 1 (1970), 195-213. MR 41 \#7202.

14. —_ Ordinary differential equations, Wiley, New York, 1971.

15. E. C. Tomastik, Singular quadratic functionals of $n$ dependent variables, Trans. Amer. Math. Soc. 124 (1966), 60-76. MR 33 \#4743.

16. Seth Warner, Modern algebra, vol. I, Prentice-Hall, Englewood Cliffs, N. J., 1965. MR 32 \#5681.

University of Missouri, Columbia, Missouri 65201 